\title{
A regularity criterion for the Navier-Stokes equations in terms of the pressure gradient
}

\author{
Stefano Bosia ${ }^{1 *}$, Monica Conti ${ }^{1 \dagger}$, Vittorino Pata ${ }^{1 \neq}$ \\ 1 Dipartimento di Matematica "F. Brioschi", Politecnico di Milano, Via Bonardi 9, 20133 Milano, Italy
}

Received 17 June 2013; accepted 19 October 2013

\section{Introduction}

Let $\Omega \subset \mathbb{R}^{3}$ be either the whole space or a bounded domain with smooth boundary $\partial \Omega$. For an arbitrarily fixed $T>0$, we consider the dimensionless form of the Navier-Stokes equations in the space-time cylinder $\Omega_{T}=\Omega \times(0, T)$

$$
\left\{\begin{array}{l}
u_{t}-\Delta u+u \cdot \nabla u+\nabla \Pi=\phi \\
\operatorname{div} u=0
\end{array}\right.
$$

The unknowns $u=u(x, t)$ and $\Pi=\Pi(x, t)$ represent the velocity vector and the pressure of a homogeneous incompressible fluid, respectively, while $\phi=\phi(x, t)$ is the density of force per unit volume. The system is complemented with the nonslip boundary condition

$$
\left.u(x, t)\right|_{x \in \partial \Omega}=0,
$$

and the initial condition

$$
u(x, 0)=u_{0}(x),
$$

for some given divergence-free function $u_{0}$ vanishing on $\partial \Omega$.

* E-mail: stefano.bosia@polimi.it

+ E-mail:monica.conti@polimi.it

‡ E-mail:vittorino.pata@polimi.it 


\subsection{Functional setting}

For $p \in[1, \infty]$ and $k \in \mathbb{N}$, the symbols $L^{p}$ and $H^{k}$ will stand for the usual Lebesgue and Sobolev spaces of real, vector or tensor valued functions on $\Omega$. The $L^{P}$-norm of a tensor valued function $w=\left\{w_{\iota}\right\}_{\iota=i j \ldots}$ is given by

$$
\left.\|w\|_{p}=\left(\int_{\Omega}|w(x)|^{p} \mathrm{~d} \boldsymbol{x}\right)^{1 / p}, \quad \text { where } \quad|w|=\sum_{\iota} w_{\iota}^{2}\right)^{1 / 2}
$$

Calling $\mathfrak{D}=\left\{u \in \mathcal{C}_{\mathrm{cpt}}^{\infty}\left(\Omega, \mathbb{R}^{3}\right): \operatorname{div} u=0\right\}$, we consider the usual Hilbert spaces associated with the Navier-Stokes equations

$$
\mathbb{H}=\text { closure of } \mathfrak{D} \text { in } L^{2}, \quad \mathbb{V}=\text { closure of } \mathfrak{D} \text { in } H^{1}, \quad \mathbb{W}=H^{2} \cap \mathbb{V} .
$$

\subsection{Regular solutions}

In what follows, we assume the initial datum $u_{0} \in \mathbb{V}$ and the external force $\phi \in L^{2}\left(0, T ; L^{2}\right)$. We begin with the classical definition (see e.g. [22]).

\section{Definition 1.1.}

A function

$$
u \in H^{1}(0, T ; \mathbb{H}) \cap \mathcal{C}([0, T], \mathbb{V}) \cap L^{2}(0, T ; \mathbb{W})
$$

is called a regular solution when equation (1) holds almost everywhere and $u(0)=u_{0}$.

Since the works of Leray [11] and Hopf [10], it is well known that for any $u_{0} \in \mathbb{H}$ (in particular, for any $u_{0} \in \mathbb{V}$ ) there exists at least a weak solution, nowadays called a Leray-Hopf solution to (1). This is a function

$$
u \in L^{\infty}(0, T ; \mathbb{H}) \cap L^{2}(0, T ; \mathbb{V})
$$

which satisfies the equation in the distributional sense, and $u(t) \rightarrow u_{0}$ weakly in $\mathbb{H}$ as $t \rightarrow 0$. At the same time, for any given $u_{0} \in \mathbb{V}$ there exists

$$
T_{\star}=T_{\star}\left(u_{0}, \phi\right) \in(0, \infty]
$$

such that (1) admits a unique regular solution $u$, provided that $T<T_{\star}$. Accordingly, the main problem in connection with Navier-Stokes equations is establishing the regularity of a Leray-Hopf solution $u$ with initial data in $\mathbb{V}$ up to the (arbitrary) time $T$. Equivalently, the goal is finding sufficient conditions in order for $u$ to be regular as well.

\subsection{Earlier results}

The question above was addressed in the fundamental works of Prodi [14] and Serrin [15] (see also [9, 17]), where $u$ is shown to be regular if

$$
u \in L^{q}\left(0, T ; L^{p}\right)
$$

for some pair $(p, q)$, where $p \in(3, \infty]$ and $q \in[2, \infty)$ fulfill the condition $3 / p+2 / q \leq 1$.

Various improvements have been subsequently obtained by several authors (see e.g. $[1,3,6,7,12,13,16,20]$ and references therein). Here, we are mainly interested in the results of [13], where the following improvement in time of (2) is provided. 
Theorem 1.2.

If there exist $\alpha \geq 0$ and a pair $(p, q)$ with $3 / p+2 / q=1$ for which

$$
\liminf _{\varepsilon \rightarrow 0} \varepsilon \int_{0}^{T-\mathrm{e}^{-1 / \varepsilon}}\|u(t)\|_{\rho}^{q(1-\alpha \varepsilon)} \mathrm{d} t<c
$$

for a suitable $c=c(\Omega, p, q, \alpha)>0$, then $u$ is the unique regular solution on $[0, T]$.

As we will see in the appendix, improving the sufficient condition of Theorem 1.2 in space is also possible, by replacing the $p$-norm with the $p(1-\alpha \varepsilon)$-norm.

Similar regularity criteria involving the gradient pressure $\nabla \Pi$ have been proposed by many authors, after the qualitative prediction in [4] that weak solutions to (1) are regular provided that

$$
\nabla \Pi \in L^{s}\left(0, T ; L^{r}\right)
$$

for a pair $(r, s)$ satisfying $3 / r+2 / s \leq 3$. The proof of this fact has been established in [2] under the restriction $s \in(1,3]$, later removed when the domain is the whole space and $\phi=0$ (see $[18,23,24])$. Weaker conditional results have been obtained in $[5,8]$ for the case $\Omega=\mathbb{R}^{3}$, in terms of pressure in Lorentz, Morrey or Besov spaces. More recently, the paper [19] improves (3) on bounded domains and for initial data $u_{0} \in L^{\infty}$, involving Lorentz spaces ${ }^{1}$ also in the time variable. The sufficient condition there reads

$$
\|\nabla \Pi\|_{L_{w}^{L}\left(0, T ; L_{w}^{r}(\Omega)\right)} \leq \varepsilon_{*}
$$

for a suitable $\varepsilon_{*}=\varepsilon_{*}(s)>0$, with $s \in(1,5 / 3)$.

\section{Main result}

The purpose of this article is to establish a novel regularity criterion in terms of the pressure gradient, valid either when $\Omega$ is bounded or $\Omega=\mathbb{R}^{3}$, and in presence of an external force $\phi \in L^{2}\left(0, T ; L^{2}\right)$. This is done in the spirit of Theorem 1.2, yielding an improved (in time) version of (3).

\section{Definition 2.1.}

A pair $(r, s)$ with $s \in(1,3]$ is called admissible if $3 / r+2 / s=3$.

Given a Leray-Hopf solution $u$ to (1) on $[0, T]$ with initial datum $u_{0} \in \mathbb{V}$, denoting $T_{\varepsilon}=T-\mathrm{e}^{-1 / \varepsilon}$, $\varepsilon>0$, our main theorem reads as follows.

\section{Theorem 2.2.}

Assume that the limit

$$
\liminf _{\varepsilon \rightarrow 0} \varepsilon^{(s-1) / 2} \int_{0}^{T_{\varepsilon}}\|\nabla \Pi(t)\|_{r}^{s(1-\varepsilon)} \mathrm{d} t=0
$$

holds for some admissible pair $(r, s)$. Then $u$ is the unique regular solution on $[0, T]$.

1 A function $v$ defined on $Q \subset \mathbb{R}^{N}$ belongs to the Lorentz space $L^{p}(Q)$-weak, denoted by $L_{\mathrm{w}}^{p}(Q)$, if

$$
\sup _{r>0} r[\mathfrak{m}\{z \in Q:|v(z)|>r\}]^{1 / p}
$$

is finite, where $\mathfrak{m}$ stands for the Lebesgue measure in $\mathbb{R}^{N}$. 
Some remarks are in order:

- With respect to the earlier literature, with particular reference to [19], we note that there exist functions satisfying limits of form (4), but which do not belong to $L_{w}^{s}\left(0, T ; L_{w}^{r}\right)$. See [13] for an example.

- Analogously to the case of Theorem 1.2, extending the sufficient condition (4) in space is also possible, replacing the $r$-norm with the $r(1-\varepsilon)$-norm. This can be easily done by recasting with minor changes the arguments of Section 4.

- A closer look at the proof shows that the conclusion of the theorem still holds if

$$
\liminf _{\varepsilon \rightarrow 0} \varepsilon^{(s-1) / 2} \int_{0}^{T_{\varepsilon}}\|\nabla \Pi(t)\|_{r}^{s(1-\varepsilon)} \mathrm{d} t<K
$$

for some constant $K=K(\Omega, r, s)>0$. In principle, such $K$ can be explicitly estimated.

- Although we work in dimension 3, our techniques apply in any dimension (by suitably rewriting the dimensiondependent inequalities).

The rest of the paper is devoted to the proof of Theorem 2.2.

\section{Preliminary facts}

\subsection{Tools and notations}

In the computations of the next sections, we will exploit the Sobolev embedding ${ }^{2}$

$$
\|u\|_{6} \leq \omega\|\nabla u\|_{2}, \quad u \in \mathbb{V}
$$

For an arbitrarily fixed $\tau \in(0, T)$, let us denote $\Omega_{\tau}=\Omega \times(0, \tau)$. Then we have the elementary interpolation

$$
\|v\|_{L^{v}\left(\Omega_{\tau}\right)} \leq\|v\|_{L^{a}\left(\Omega_{\tau}\right)}^{\sigma}\|v\|_{L^{b}\left(\Omega_{\tau}\right)^{\prime}}^{1-\sigma}
$$

for all $1 \leq a \leq b \leq \infty$ and $a \leq \gamma \leq b$ such that $1 / \gamma=\sigma / a+(1-\sigma) / b$. We shall also make use of the embedding $L^{\infty}\left(0, \tau ; L^{2}\right) \cap L^{2}\left(0, \tau ; L^{6}\right) \subset L^{b}\left(0, \tau ; L^{a}\right)$, valid (in dimension 3 ) for all $2 \leq a \leq 6$ and $2 \leq b \leq \infty$ satisfying $3 / a+2 / b=3 / 2$. In particular, the corresponding interpolation estimate reads

$$
\|v\|_{a, b} \leq\|v\|_{2, \infty}^{1-\sigma}\|v\|_{6,2}^{\sigma} \quad \text { with } \quad \sigma=\frac{2}{b}
$$

where, here and in the sequel, we write for short

$$
\|v\|_{a, b}= \begin{cases}\left(\int_{0}^{\tau}\|v(t)\|_{a}^{b} \mathrm{~d} t\right)^{1 / b} & \text { if } \quad b<\infty \\ \underset{\substack{\operatorname{ess} \sup \\ t \in[0, \tau]}}{ }\|v(t)\|_{a} & \text { if } \quad b=\infty .\end{cases}
$$

An elementary remark will be needed.

2 According to [21], we have $\omega=(2 / \pi)^{2 / 3} / \sqrt{3}$. 


\section{Lemma 3.1.}

Let $x_{\varepsilon} \in \mathbb{R}$ satisfy $\liminf _{\varepsilon \rightarrow 0} \varepsilon x_{\varepsilon}^{a}=0$ for some $a>0$. Then, for every fixed $\eta>0, \liminf _{\varepsilon \rightarrow 0} \varepsilon x_{\varepsilon}^{a+\eta \varepsilon}=0$.

Proof. By assumption, there is $\varepsilon_{n} \rightarrow 0$ such that $\varepsilon_{n} x_{\varepsilon_{n}}^{a} \rightarrow 0$. Thus, for $n$ large, $\log x_{\varepsilon_{n}} \leq-(1 / a) \log \varepsilon_{n}$. Accordingly,

$$
\varepsilon_{n} x_{\varepsilon_{n}}^{a+n \varepsilon_{n}}=\varepsilon_{n} x_{\varepsilon_{n}}^{a} \mathrm{e}^{n \varepsilon_{n} \log x_{\varepsilon_{n}}} \leq \varepsilon_{n} x_{\varepsilon_{n}}^{a} \mathrm{e}^{-\left(\eta \varepsilon_{n} / a\right) \log \varepsilon_{n}} \rightarrow 0
$$

proving the claim.

The next well-known identity can be verified by direct computations.

\section{Lemma 3.2.}

Let $u: \Omega \rightarrow \mathbb{R}^{3}$ be a vector field satisfying div $u=0$ and $\left.u\right|_{\partial \Omega}=0$. Then

$$
-\int_{\Omega} \Delta u \cdot u|u|^{\beta-2} \mathrm{~d} x=\int_{\Omega}|u|^{\beta-2}|\nabla u|^{2}+\left.\left.\frac{4(\beta-2)}{\beta^{2}} \int_{\Omega}|\nabla| u\right|^{\beta / 2}\right|^{2} \mathrm{~d} x
$$

for all

$\geq 2$.

\subsection{From $\nabla \Pi$-estimates to $u$-estimates}

Throughout the paper, we agree to denote

$$
\Phi=\left(\int_{0}^{T}\|\phi(t)\|_{2}^{2} \mathrm{~d} t\right)^{1 / 2}<\infty
$$

Let $u$ be a Leray-Hopf solution to (1) on $[0, T]$ with $u_{0} \in \mathbb{V}$, and let $\tau \in(0, T)$ be arbitrarily fixed.

\section{Proposition 3.3.}

Let $\theta \in[5 / 2,6]$ be given ${ }^{3}$, and let $(r, s)$ be any pair satisfying

$$
\frac{3}{r}+\frac{2}{s}=2+\frac{3}{\theta} \quad \text { with } \quad s \in(1, \theta] .
$$

Then, for the pair $(p, q)$ given by

$$
p=\frac{r(\theta-1)}{r-1}, \quad q=\frac{s(\theta-1)}{s-1}
$$

we have the estimate

$$
\int_{0}^{\tau}\|u(t)\|_{p}^{q} \mathrm{~d} t \leq C\left\|u_{0}\right\|_{\theta}^{q}+C \Phi^{q}+C\left(\int_{0}^{\tau}\|\nabla \Pi(t)\|_{r}^{s} \mathrm{~d} t\right)^{q / s}
$$

Here, $C>0$ is independent of $\tau$ and $\theta$.

3 The lower bound 5/2 is assumed in order to have constants independent of $\theta$. 
Proof. Following the argument in [18], we multiply equation (1) by $u|u|^{\theta-2}$. Exploiting Lemma 3.2 we obtain

$$
\frac{1}{\theta} \frac{\mathrm{d}}{\mathrm{d} t}\|u\|_{\theta}^{\theta}+\int_{\Omega}|\nabla u|^{2}|u|^{\theta-2} \mathrm{~d} x+\left.\left.\frac{4(\theta-2)}{\theta^{2}} \int_{\Omega}|\nabla| u\right|^{\theta / 2}\right|^{2} \mathrm{~d} x=\int_{\Omega} \phi u|u|^{\theta-2} \mathrm{~d} x+\mathfrak{I}
$$

having set

$$
\mathfrak{I}=-\int_{\Omega} u \cdot \nabla \Pi|u|^{\theta-2} \mathrm{~d} x
$$

Calling $v=|u|^{\theta / 2}$, the latter identity turns into

$$
\frac{1}{\theta} \frac{\mathrm{d}}{\mathrm{d} t}\|v\|_{2}^{2}+\frac{4(\theta-2)}{\theta^{2}}\|\nabla v\|_{2}^{2}=\int_{\Omega} \phi u|u|^{\theta-2} \mathrm{~d} x+\mathfrak{I}
$$

and an integration on $\left(0, \tau^{\prime}\right)$ with $\tau^{\prime} \leq \tau$ yields

$$
\frac{1}{\theta}\|v\|_{2, \infty}^{2}+\frac{4(\theta-2)}{\theta^{2}}\|\nabla v\|_{2,2}^{2} \leq \frac{1}{\theta}\|v(0)\|_{2}^{2}+\int_{0}^{\tau} \int_{\Omega} \phi u|u|^{\theta-2} \mathrm{~d} x \mathrm{~d} t+\int_{0}^{\tau} \mathfrak{I} \mathrm{d} t .
$$

We observe that

$$
\int_{0}^{\tau} \int_{\Omega} \phi u|u|^{\theta-2} \mathrm{~d} x \mathrm{~d} t \leq \Phi\|u\|_{2(\theta-1), 2(\theta-1)}^{\theta-1} \leq \Phi\|v\|_{4(\theta-1) / \theta, 4(\theta-1) / \theta}^{2(\theta-1) / \theta} .
$$

The norm of $v$ appearing in the inequality can be estimated in terms of $\|v\|_{2, \infty}$ and $\|\nabla v\|_{2,2}$. Indeed, applying (5) with $\gamma=4(\theta-1) / \theta, a=2$ and $b=10 / 3$, we obtain

$$
\|v\|_{4(\theta-1) / \theta, 4(\theta-1) / \theta} \leq\|v\|_{2,2}^{(6-\theta) / 4(\theta-1)}\|v\|_{10 / 3,10 / 3}^{5(\theta-2) / 4(\theta-1)}
$$

Since from (6) with $a=b=10 / 3,\|v\|_{10 / 3,10 / 3} \leq\|v\|_{2, \infty}^{2 / 5}\|v\|_{6,2}^{3 / 5}$, exploiting the elementary control $\|v\|_{2,2} \leq \tau^{1 / 2}\|v\|_{2, \infty} \leq$ $T^{1 / 2}\|v\|_{2, \infty}$, we draw the following chain of inequalities:

$$
\|v\|_{4(\theta-1) / \theta, 4(\theta-1) / \theta} \leq\|v\|_{2,2}^{(6-\theta) / 4(\theta-1)}\|v\|_{10 / 3,10 / 3}^{5(\theta-2) / 4(\theta-1)} \leq T^{(6-\theta) / 8(\theta-1)}\|v\|_{2, \infty}^{(\theta+2) / 4(\theta-1)}\|v\|_{6,2}^{3(\theta-2) / 4(\theta-1)} .
$$

By the Sobolev embedding and a suitable use of the Young inequality, we arrive at

$$
\int_{0}^{\tau} \int_{\Omega} \phi u|u|^{\theta-2} \mathrm{~d} x \mathrm{~d} t \leq C_{T} \Phi^{\theta}+\frac{1}{2 \theta}\|v\|_{2, \infty}^{2}+\frac{2(\theta-2)}{\theta^{2}}\|\nabla v\|_{2,2}^{2}
$$

where $C_{T}>0$ is a positive constant independent of $\tau$ and $\theta$. In light of the estimates above, we deduce from (8)

$$
\frac{1}{2 \theta}\|v\|_{2, \infty}^{2}+\frac{2(\theta-2)}{\theta^{2}}\|\nabla v\|_{2,2}^{2} \leq \frac{1}{\theta}\|v(0)\|_{2}^{2}+C_{T} \Phi^{\theta}+\int_{0}^{\tau} \mathfrak{I} \mathrm{d} t .
$$

In order to bound the term containing $\mathfrak{I}$, we proceed as follows:

$$
\int_{0}^{\tau} \mathfrak{I} \mathrm{d} t \leq \int_{0}^{\tau} \int_{\Omega}\left|\nabla \Pi\left\|\left.u\right|^{\theta-1} \mathrm{~d} x \mathrm{~d} t \leq\right\| \nabla \Pi\left\|_{r, s}\right\| u\left\|_{(\theta-1) r^{*},(\theta-1) s^{*}}^{\theta-1} \leq\right\| \nabla \Pi\left\|_{r, s}\right\| v \|_{2 p / \theta, 2 q / \theta}^{2(\theta-1) / \theta}\right.
$$

where $r^{*}=r /(r-1)$ and $s^{*}=s /(s-1)$ are the Hölder conjugates of $r$ and $s$, respectively, and $p=(\theta-1) r^{*}, q=(\theta-1) s^{*}$. We now note that $2<2 p / \theta \leq 6,2 \leq 2 q / \theta<\infty$, and, recalling (7),

$$
\frac{3}{2 p / \theta}+\frac{2}{2 q / \theta}=\frac{3}{2}
$$


Therefore, we are in the position to apply (6), to get

$$
\|v\|_{2 p / \theta, 2 q / \theta}^{2} \leq\|v\|_{2, \infty}^{2(1-\sigma)}\|v\|_{6,2}^{2 \sigma}
$$

where $\sigma=\theta / q$. Hence, the Sobolev embedding and the Young inequality for the conjugate exponents $1 /(1-\sigma), 1 / \sigma$ yield

$$
\varkappa\|v\|_{2 p / \theta, 2 q / \theta}^{2} \leq \varkappa(1-\sigma) c^{1 /(1-\sigma)}\|v\|_{2, \infty}^{2}+\varkappa \sigma c^{-1 / \sigma} \omega^{2}\|\nabla v\|_{2,2}^{2}
$$

for all $\varkappa>0$ and $c>0$. By fixing in a proper way $\varkappa$ and $c$, we get

$$
\varkappa\|v\|_{2 p / \theta, 2 q / \theta}^{2} \leq \frac{1}{2 \theta}\|v\|_{2, \infty}^{2}+\frac{2(\theta-2)}{\theta^{2}}\|\nabla v\|_{2,2}^{2},
$$

and, in light of (9), we draw the conclusion

$$
\varkappa\|v\|_{2 p / \theta, 2 q / \theta}^{2} \leq \frac{1}{\theta}\|v(0)\|_{2}^{2}+C_{T} \Phi^{\theta}+\|\nabla \Pi\|_{r, s}\|v\|_{2 p / \theta, 2 q / \theta}^{2(\theta-1) / \theta} .
$$

By a further use of the Young inequality,

$$
\|\nabla \Pi\|_{r, s}\|v\|_{2 p / \theta, 2 q / \theta}^{2(\theta-1) / \theta} \leq \frac{\varkappa}{2}\|v\|_{2 p / \theta, 2 q / \theta}^{2}+C_{\varkappa}\|\nabla \Pi\|_{r, s}^{\theta}
$$

for some $C_{\varkappa}>0$, yielding the final relation

$$
\frac{\varkappa}{2}\|v\|_{2 p \mid \theta, 2 q / \theta}^{2} \leq \frac{1}{\theta}\|v(0)\|_{2}^{2}+C_{T} \Phi^{\theta}+C_{\varkappa}\|\nabla \Pi\|_{r, s}^{\theta} .
$$

Written in terms of $u$, this is

$$
\frac{\varkappa}{2}\|u\|_{p, q}^{\theta} \leq \frac{1}{\theta}\left\|u_{0}\right\|_{\theta}^{\theta}+C_{T} \Phi^{\theta}+C_{\varkappa}\|\nabla \Pi\|_{r, s}^{\theta}
$$

as claimed.

\section{Proof of main result}

Let $\tau \in(0, T)$ be arbitrarily fixed, a nd let $\varepsilon>0$ be sufficiently s mall. A long the p roof, $c>0$ will denote a generic constant, independent of $\tau$ and $\varepsilon$, which may change even from line to line. Let $(r, s)$ be an admissible pair for which (4) holds true. Defining $s_{\varepsilon}=(1-\varepsilon) s$, the couple $\left(r, s_{\varepsilon}\right)$ is e asily seen to satisfy the relation

$$
\frac{3}{r}+\frac{2}{s_{\varepsilon}}=2+\frac{3}{\theta_{\varepsilon}}, \quad \text { where } \quad \theta_{\varepsilon}=\frac{3(1-\varepsilon) s}{(1-\varepsilon) s+2 \varepsilon} .
$$

Since $\theta_{\varepsilon} \uparrow 3$ as $\varepsilon \rightarrow 0$, up to choosing $\varepsilon>0$ sufficiently small,

$$
s \in(1,3] \quad \Longrightarrow \quad s_{\varepsilon} \in\left(1, \theta_{\varepsilon}\right]
$$

Therefore, we can apply Proposition 3.3 with $\theta=\theta_{\varepsilon}$ and the pair $\left(r, s_{\varepsilon}\right)$. This entails

$$
\int_{0}^{\tau}\|u\|_{p_{\varepsilon}}^{q_{\varepsilon}} \mathrm{d} t \leq C\left\|u_{0}\right\|_{\theta_{\varepsilon}}^{q_{\varepsilon}}+C \Phi^{q_{\varepsilon}}+C\left(\int_{0}^{\tau}\|\nabla \Pi\|_{r}^{s_{\varepsilon}} \mathrm{d} t\right)^{q_{\varepsilon} / s_{\varepsilon}}
$$


where $p_{\varepsilon}=\left(\theta_{\varepsilon}-1\right) r^{*}, q_{\varepsilon}=\left(\theta_{\varepsilon}-1\right) s_{\varepsilon}^{*}$, again, the star is the Hölder conjugate. It is worth noting that the pair $(p, q)$ given by $p=2 r^{*}, q=2 s^{*}$, fulfills the identity

$$
\frac{3}{p}+\frac{2}{q}=1
$$

Since by assumption $s \in(1,3]$, it is readily seen that

$$
p>3
$$

We rewrite $p_{\varepsilon}$ in the form

$$
p_{\varepsilon}=\left(\theta_{\varepsilon}-1\right) r^{*}=p\left(1-\alpha_{\varepsilon} \varepsilon\right) \quad \text { with } \quad \alpha_{\varepsilon}=\frac{3}{(1-\varepsilon) s+2 \varepsilon} .
$$

Analogous computations provide

$$
q_{\varepsilon}=q\left(1-\alpha_{\varepsilon} \varepsilon\right)\left(1+\frac{\varepsilon}{s(1-\varepsilon)-1}\right) .
$$

We are now ready to conclude the proof of Theorem 2.2. Indeed, setting

$$
\alpha=\frac{3}{2} \lim _{\varepsilon \rightarrow 0} \alpha_{\varepsilon}=\frac{9}{2 s}
$$

in order to ensure $2<p(1-\alpha \varepsilon)<p_{\varepsilon}$, we use the interpolation estimate

$$
\|u\|_{p(1-\alpha \varepsilon)} \leq\|u\|_{2}^{1-\sigma_{\varepsilon}}\|u\|_{p_{\varepsilon}}^{\sigma_{\varepsilon}}
$$

valid for a suitable $\sigma_{\varepsilon} \in(0,1)$. Since $q(1-\alpha \varepsilon) \sigma_{\varepsilon}<q_{\varepsilon}$, a standard application of the Young inequality gives

$$
\int_{0}^{\tau}\|u\|_{p(1-\alpha \varepsilon)}^{q(1-\alpha \varepsilon)} \mathrm{d} t \leq \int_{0}^{\tau}\|u\|_{2}^{q(1-\alpha \varepsilon)\left(1-\sigma_{\varepsilon}\right)}\|u\|_{p_{\varepsilon}}^{q(1-\alpha \varepsilon) \sigma_{\varepsilon}} \mathrm{d} t \leq R_{0}+\int_{0}^{\tau}\|u\|_{p_{\varepsilon}}^{q_{\varepsilon}} \mathrm{d} t
$$

for some positive constant $R_{0}$ depending explicitly (besides on $T$ ) on the quantity $\|u\|_{2, \infty}$, which is known to be bounded, with a bound depending on the initial datum $u_{0}$.

At this point, we make the choice $\tau=T_{\varepsilon}$. Then, collecting (10) and (13), we are led to

$$
\begin{aligned}
\liminf _{\varepsilon \rightarrow 0} \varepsilon \int_{0}^{T_{\varepsilon}}\|u\|_{p(1-\alpha \varepsilon)}^{q(1-\alpha \varepsilon)} \mathrm{d} t & \leq c \liminf _{\varepsilon \rightarrow 0} \varepsilon \int_{0}^{T_{\varepsilon}}\|u\|_{p_{\varepsilon}}^{q_{\varepsilon}} \mathrm{d} t \\
& \leq c \liminf _{\varepsilon \rightarrow 0} \varepsilon\left[\left\|u_{0}\right\|_{\theta_{\varepsilon}}^{q_{\varepsilon}}+\Phi^{q_{\varepsilon}}+\left(\int_{0}^{T_{\varepsilon}}\|\nabla \Pi\|_{r}^{s_{\varepsilon}} \mathrm{d} t\right)^{q_{\varepsilon} / s_{\varepsilon}}\right]=c \liminf _{\varepsilon \rightarrow 0} \varepsilon\left(\int_{0}^{T_{\varepsilon}}\|\nabla \Pi\|_{r}^{s_{\varepsilon}} \mathrm{d} t\right)^{q_{\varepsilon} / s_{\varepsilon}} .
\end{aligned}
$$

It is easily seen that

$$
\frac{2}{s-1} \leq \frac{q_{\varepsilon}}{s_{\varepsilon}} \leq \frac{2}{s-1}+\eta \varepsilon
$$

for some finite $\eta>0$, depending only on s. Hence, exploiting Lemma 3.1 jointly with (4), we have

$$
\liminf _{\varepsilon \rightarrow 0} \varepsilon\left(\int_{0}^{T_{\varepsilon}}\|\nabla \Pi\|_{r}^{s_{\varepsilon}} \mathrm{d} t\right)^{q_{\varepsilon} / s_{\varepsilon}}=\liminf _{\varepsilon \rightarrow 0} \varepsilon\left(\int_{0}^{T_{\varepsilon}}\|\nabla \Pi\|_{r}^{s_{\varepsilon}} \mathrm{d} t\right)^{2 /(s-1)}=0 .
$$

In light of the above computations we conclude that

$$
\liminf _{\varepsilon \rightarrow 0} \varepsilon \int_{0}^{T_{\varepsilon}}\|u\|_{p(1-\alpha \varepsilon)}^{q(1-\alpha \varepsilon)} \mathrm{d} t=0
$$

for $\alpha>0$ and the pair $(p, q)$ above, which meets the requirements of Theorem A.1, see (11)-(12). As a consequence of its application, we eventually learn that $u$ is the (unique) regular solution on $[0, T]$. This finishes the proof. 


\section{Appendix}

We extend the main Theorem 5.1 from [13], providing a Prodi-Serrin type criterion requiring a weaker condition in space for the norm of $u$. We first introduce some n otation. We introduce the Stokes operator $A=-P \Delta$ with domain $\mathbb{W}$, where $P: L^{2} \rightarrow \mathbb{H}$ is the Leray-Helmholtz orthogonal projection, and we denote by $\kappa=\kappa(\Omega)>0$ the best constant such that ${ }^{4}$

$$
\|\nabla u\|_{6} \leq \kappa\|A u\|, \quad u \in \mathbb{W} .
$$

In what follows, let $u$ be a fixed Leray-Hopf solution to (1) with $u(0)=u_{0} \in \mathbb{V}$. Besides, let $(p, q)$ denote a pair, $p \in(3, \infty], q \in[2, \infty)$, subject to the condition

$$
\frac{3}{p}+\frac{2}{q}=1
$$

Defining the function of the variable $\omega \geq 0$

$$
H(\omega)= \begin{cases}\frac{1-\mathrm{e}^{-\omega}}{\omega} & \text { if } \quad \omega>0 \\ 1 & \text { if } \quad \omega=0\end{cases}
$$

and the positive constant

$$
C_{q}=\frac{q^{q}}{4 \kappa^{q-2}(q-1)^{q-1}}
$$

the result reads as follows (recall that $T_{\varepsilon}=T-\mathrm{e}^{-1 / \varepsilon}$ ).

\section{Theorem A.1.}

If there exist $\alpha \geq 0$ and a pair $(p, q)$ for which

$$
\liminf _{\varepsilon \rightarrow 0} \varepsilon \int_{0}^{T_{\varepsilon}}\|u(t)\|_{p(1-\alpha \varepsilon)}^{q(1-\alpha \varepsilon)} \mathrm{d} t<H(q \alpha) C_{q},
$$

then $u$ is the unique regular solution on $[0, T]$.

The proof of Theorem A.1 is carried out along the lines of [13]. The key ingredient is a refined version of Lemma 9.3 therein.

\section{Lemma A.2.}

Let $f=P \phi \in L^{2}(0, T ; \mathbb{H})$, and let $(p, q)$ and $\delta \in(0,1)$ be fixed. For every $\sigma>0$ sufficiently small, we have

$$
\frac{\mathrm{d}}{\mathrm{d} t}\|\nabla u\|^{2} \leq \frac{\mu(\sigma)}{2(1-\delta)^{q-1} C_{q}}\|u\|_{\rho(1-\sigma)}^{q(1-\sigma)}\|\nabla u\|^{2+2 q \sigma}+\frac{1}{2 \delta}\|f\|^{2},
$$

for some nonnegative function $\mu$ (depending on $p, q, \delta, \Omega$ ) satisfying $\lim _{\sigma \rightarrow 0} \mu(\sigma)=1$.

${ }^{4}$ For the case $\Omega=\mathbb{R}^{3}$ we have the explicit value $\kappa=(2 / \pi)^{2 / 3} / \sqrt{3}$ (see [13, Appendix]). 
Proof. According to [13, Lemma 9.2], for every pair $(p, q)$ and every $\delta \in(0,1)$, we have the inequality

$$
\frac{\mathrm{d}}{\mathrm{d} t}\|\nabla u\|^{2} \leq \frac{1}{2(1-\delta)^{q-1} C_{q}}\|u\|_{p}^{q}\|\nabla u\|^{2}+\frac{1}{2 \delta}\|f\|^{2}
$$

We now consider (A.4) with $(p, q)$ replaced by the pair $\left(p_{\sigma}, q_{\sigma}\right)$ given by

$$
p_{\sigma}=\frac{3 p(1+\sigma)}{3+\sigma p}, \quad q_{\sigma}=q(1+\sigma)
$$

Calling $\vartheta=2 \sigma /(1+\sigma)$, we obtain by interpolation

$$
\|u\|_{p_{\sigma}}^{q_{\sigma}} \leq\|u\|_{p(1-\sigma)}^{(1-\vartheta) q_{\sigma}}\|u\|_{6}^{\vartheta q_{\sigma}}=\|u\|_{p(1-\sigma)}^{q(1-\sigma)}\|u\|_{6}^{2 q \sigma} \leq c^{\sigma}\|u\|_{p(1-\sigma)}^{q(1-\sigma)}\|\nabla u\|^{2 q \sigma}
$$

for some $c=c(\Omega)>0$. Therefore,

$$
\frac{1}{2(1-\delta)^{q_{\sigma}-1} C_{q_{\sigma}}}\|u\|_{p_{\sigma}}^{q_{\sigma}}\|\nabla u\|^{2} \leq \frac{\mu(\sigma)}{2(1-\delta)^{q-1} C_{q}}\|u\|_{\rho(1-\sigma)}^{q(1-\sigma)}\|\nabla u\|^{2+2 q \sigma},
$$

where the function

$$
\mu(\sigma)=\frac{c^{\sigma} C_{q}}{(1-\delta)^{q \sigma} C_{q_{\sigma}}}
$$

is easily seen to fulfill the required limit.

Denoting now

$$
L=\liminf _{\varepsilon \rightarrow 0} \varepsilon \int_{0}^{T_{\varepsilon}}\|u(t)\|_{p(1-\alpha \varepsilon)}^{q(1-\alpha \varepsilon)} \mathrm{d} t
$$

we know by assumption that $L<H(q \alpha) C_{q}$. Calling for short $\varphi=\|\nabla u\|^{2}$ and fixing $\delta=\delta(q)>0$ small enough in order to satisfy

$$
\beta=\frac{L}{2(1-\delta)^{q-1} C_{q}}<\frac{1}{2} H(q \alpha)
$$

we deduce from (A.3) the differential inequality

$$
\varphi^{\prime} \leq \frac{\beta \mu(\sigma)}{L}\|u\|_{p(1-\sigma)}^{q(1-\sigma)} \varphi^{1+q \sigma}+\frac{1}{2 \delta}\|f\|^{2}
$$

valid for any $\sigma>0$ small. At this point, assuming $\varepsilon$ sufficiently small, we choose $\sigma=\alpha \varepsilon$. Then, introducing the family of functions

$$
\lambda_{\varepsilon}(t)=\frac{\beta \mu(\alpha \varepsilon)}{L}\|u(t)\|_{p(1-\alpha \varepsilon)}^{q(1-\alpha \varepsilon)}
$$

the inequality above reads

$$
\varphi^{\prime} \leq \lambda_{\varepsilon} \varphi^{1+q \alpha \varepsilon}+\frac{1}{2 \delta}\|f\|^{2}
$$

Integrating on $[\tau, t]$, we obtain

$$
\varphi(t) \leq \varphi(\tau)+\int_{\tau}^{t} \lambda_{\varepsilon}(s)[\varphi(s)]^{1+q \alpha \varepsilon} \mathrm{d} s+\frac{1}{2 \delta} \int_{\tau}^{t}\|f(s)\|^{2} \mathrm{~d} s
$$

with

$$
\liminf _{\varepsilon \rightarrow 0} \varepsilon \int_{0}^{T_{\varepsilon}} \lambda_{\varepsilon}(t) \mathrm{d} t=\beta<\frac{1}{2} H(q \alpha) .
$$

Then, by applying [13, Lemma 9.1], we deduce the limit

$$
\liminf _{t \rightarrow T} \sqrt{T-t} \varphi(t)=0
$$

This is incompatible with the blow-up of $\|\nabla u\|$ at $T$ (cf. [13, Lemma 9.4]). The proof of Theorem A.1 is finished. 


\section{References}

[1] Beirão da Veiga $H$., Remarks on the smoothness of the $L^{\infty}\left(0, T ; L^{3}\right)$ solutions of the 3-D Navier-Stokes equations, Portugal. Math., 1997, 54(4), 381-391

[2] Berselli L.C., Galdi G.P., Regularity criteria involving the pressure for the weak solutions to the Navier-Stokes equations, Proc. Amer. Math. Soc., 2002, 130(12), 3585-3595

[3] Bjorland C., Vasseur A., Weak in space, log in time improvement of the Ladyženskaja-Prodi-Serrin criteria, J. Math. Fluid Mech., 2011, 13(2), 259-269

[4] Caffarelli L., Kohn R., Nirenberg L., Partial regularity of suitable weak solutions of the Navier-Stokes equations, Comm. Pure Appl. Math., 1982, 35(6), 771-831

[5] Cai Z., Fan J., Zhai J., Regularity criteria in weak spaces for 3-dimensional Navier-Stokes equations in terms of the pressure, Differential Integral Equations, 2010, 23(11-12), 1023-1033

[6] Chan C.H., Vasseur A., Log improvement of the Prodi-Serrin criteria for Navier-Stokes equations, Methods Appl. Anal., 2007, 14(2), 197-212

[7] Escauriaza L., Seregin G., Šverák V., $L_{3, \infty}$-solutions of the Navier-Stokes equations and backward uniqueness, Russian Math. Surveys, 2003, 58(2), 211-250

[8] Fan J., Jiang S., Ni G., On regularity criteria for the $n$-dimensional Navier-Stokes equations in terms of the pressure, J. Differential Equations, 2008, 244(11), 2963-2979

[9] Giga Y., Solutions for semilinear parabolic equations in $L^{p}$ and regularity of weak solutions of the Navier-Stokes equations, J. Differential Equations, 1986, 62(2), 186-212

[10] Hopf E., Über die Anfangswertaufgabe für die hydrodynamischen Grundgleichungen, Math. Nachr., 1951, 4, 213-231

[11] Leray J., Sur le mouvement d'un liquide visqueux emplissant l'espace, Acta Math., 1934, 63, 193-248

[12] Montgomery-Smith S., Conditions implying regularity of the three dimensional Navier-Stokes equation, Appl. Math., 2005, 50(5), 451-464

[13] Pata V., On the regularity of solutions to the Navier-Stokes equations, Commun. Pure Appl. Anal., 2012, 11(2), 747-761

[14] Prodi G., Un teorema di unicità per le equazioni di Navier-Stokes, Ann. Mat. Pura Appl., 1959, 48, 173-182

[15] Serrin J., On the interior regularity of weak solutions of the Navier-Stokes equations, Arch. Rational Mech. Anal., 1962, 9, 187-195

[16] Sohr H., A regularity class for the Navier-Stokes equations in Lorentz spaces, J. Evol. Equ., 2001, 1(4), 441-467

[17] Struwe M., On partial regularity results for the Navier-Stokes equations, Comm. Pure Appl. Math., 1988, 41(4), 437-458

[18] Struwe M., On a Serrin-type regularity criterion for the Navier-Stokes equations in terms of the pressure, J. Math. Fluid Mech., 2007, 9(2), 235-242

[19] Suzuki T., Regularity criteria of weak solutions in terms of the pressure in Lorentz spaces to the Navier-Stokes equations, J. Math. Fluid Mech., 2012, 14(4), 653-660

[20] Takahashi S., On interior regularity criteria for weak solutions of the Navier-Stokes equations, Manuscripta Math., 1990, 69(3), 237-254

[21] Talenti G., Best constant in Sobolev inequality, Ann. Mat. Pura Appl., 1976, 110, 353-372

[22] Temam R., Navier-Stokes Equations, AMS Chelsea, Providence, 2001

[23] Zhou Y., On regularity criteria in terms of pressure for the Navier-Stokes equations in $\mathbb{R}^{3}$, Proc. Amer. Math. Soc., 2006, 134(1), 149-156

[24] Zhou Y., On a regularity criterion in terms of the gradient of pressure for the Navier-Stokes equations in $\mathbb{R}^{N}$, Z. Angew. Math. Phys., 2006, 57(3), 384-392 